Characterization of the SIDDHARTA-2 second level trigger detector prototype based on scintillators coupled to a prism reflector light guide

This content has been downloaded from IOPscience. Please scroll down to see the full text. 2013 JINST 8 T11003

(http://iopscience.iop.org/1748-0221/8/11/T11003)

View the table of contents for this issue, or go to the journal homepage for more

Download details:

IP Address: 61.129.42.30

This content was downloaded on 18/11/2016 at 06:49

Please note that terms and conditions apply.

You may also be interested in:

Characterization of a scintillating fibers read by MPPC detectors trigger prototype for the AMADEUS

experiment

M Bazzi, C Berucci, C Curceanu et al.

Kaonic helium X-ray measurement in the SIDDHARTA experiment

H Shi, M Bazzi, G Beer et al.

Progress and perspectives in the low-energy kaon-nucleon/nuclei interaction studies at the DANE

collider

M lliescu, M Bazzi, G Beer et al.

Quartz Cherenkov counters for fast timing: QUARTIC

M G Albrow, Heejong Kim, S Los et al.

Kaonic atoms measurements at the DANE collider: the SIDDHARTA experiment

A Rizzo, M Bazzi, G Beer et al.

The high timing resolution T0 system in IHEP E3 beam line

S Qian, Y Wang, Z Ning et al. 


\section{Characterization of the SIDDHARTA-2 second level trigger detector prototype based on scintillators coupled to a prism reflector light guide}

M. Bazzi, ${ }^{a}$ C. Berucci, ${ }^{b}$ C. Curceanu, ${ }^{a}$ A. d'Uffizi, ${ }^{a}{ }^{a}$ M. Iliescu, ${ }^{a}$ E. Sbardella, ${ }^{a}$ A. Scordo, ${ }^{a}$ H. Shi, ${ }^{b}$ F. Sirghi, ${ }^{a, c}$ H. Tatsuno ${ }^{a}$ and I. Tucakovic ${ }^{a, 1}$

${ }^{a}$ Laboratori Nazionali di Frascati, INFN,

Via E. Fermi 40, Frascati (Rome), Italy

${ }^{b}$ Stefan-Meyer-Institut für subatomare Physik, Boltzmanngasse 3, Vienna, Austria

${ }^{c}$ Institutul National pentru Fizica si Inginerie Nucleara Horia Hulubei, IFIN-HH, Reactorului 30, Magurele, Bucharest, Romania

E-mail: ivana.tucakovic@lnf.infn.it

ABSTRACT: The SIDDHARTA experiment at the DAФNE collider of LNF-INFN performed in 2009 high precision measurements of kaonic hydrogen and kaonic helium atomic transitions. To determine the $\bar{K} N$ isospin dependent scattering lenghts an important measurement, namely the kaonic deuterium one, is, however, still missing. Due to the very low expected yield of the kaonic deuterium $K_{\alpha}$ transition, a major improvement in the signal over background ratio is needed. To achieve a further background reduction, a second level trigger, based on the detection of charged pions produced by the $\mathrm{K}^{-}$absorption on various materials, including the target gas nuclei, is planned to be implemented in the future SIDDHARTA-2 experiment. For shielding-related geometrical limitations, a single side of the scintillators can be accessed; in order to reach a good time resolution and uniform efficiency, a both-end readout was then realized with complex multi-reflection light guides. In this work, the results of the tests made on a detector prototype, performed on the $\pi M-1$ beamline of the Paul Scherrer Institute (Switzerland), are presented. The tests had the goal to determine the efficiency and the time resolution for pions, which should comply with the minimum required values of $90 \%$ and $1 \mathrm{~ns}$ (FWHM) respectively. The obtained results, $96 \%$ efficiency and $750 \mathrm{ps}$ FWHM for $170 \mathrm{MeV} / \mathrm{c}$ momentum pions, qualify the prototype as an excellent second level trigger for the SIDDHARTA-2 experiment. Similar results for $170 \mathrm{MeV} / \mathrm{c}$ momentum muons and electrons are also presented.

KEYWORDS: Trigger concepts and systems (hardware and software); Scintillators, scintillation and light emission processes (solid, gas and liquid scintillators); Scintillators and scintillating fibres and light guides; Trigger detectors

\footnotetext{
${ }^{1}$ Corresponding author.
} 


\section{Contents}

1 Introduction 1

2 Monte Carlo simulations 2

2.1 Full setup Monte Carlo simulations 2

2.2 Second level trigger dedicated study 4

3 Second level trigger prototype test 5

4 Beam test results $\quad 7$

4.1 DAQ chain $\quad 7$

$\begin{array}{lll}4.2 & \text { Particles identification and events selection } & 7\end{array}$

$\begin{array}{lll}4.3 & \text { Time resolution results } & 7\end{array}$

$\begin{array}{lll}4.4 & \text { Efficiency results } & 10\end{array}$

5 Higher momentum test results $\quad 10$

6 Conclusions 10

\section{Introduction}

The SIDDHARTA experiment measured in 2009 the $K$-series X-ray transitions of kaonic hydrogen $(\mathrm{KH})$ atoms $[1,2]$ and the $L$-series ones of kaonic ${ }^{3,4} \mathrm{He}[3,4]$ at the DAФNE $e^{+} e^{-}$collider of Laboratori Nazionali di Frascati (LNF) of INFN. The provided values for the shift and the width of the $1 \mathrm{~s}$ and, respectively, $2 p$ levels, induced by the presence of the strong interaction between the kaon and nucleus with respect to the purely QED calculated values, are intensively used by theoreticians [5-7]. Further theoretical studies require the measurement of the kaonic deuterium (KD) $K$-series transitions that, together with the $\mathrm{KH}$ ones, will allow the extraction of the isospin-dependent $\bar{K} N$ scattering lengths, fundamental for understanding the low-energy QCD in the strangeness sector [8]. The $K^{-} D$ measurement was not yet performed due to the very low yields of the involved transitions, estimated to be $\sim 10^{-3}$ by an exploratory SIDDHARTA measurement [9] and by theoretical calculations [10]. This measurement will be the main goal of the SIDDHARTA-2 experiment [11]. In order to deal with the low expected signal and the large amount of background, an important aspect is to select the events occuring only in the target gas $\left(D_{2}\right)$, thus eliminating the hadronic and X-ray background corresponding to kaons stopped elsewhere (degrader, entrance windows, frames, etc.). Since shielding requirements do not allow surrounding the target with a tracker, the SIDDHARTA-2 strategy consists in identifying events inside the gas by their characteristic timing, related to kaon moderation. In SIDDHARTA-2, the slowing-down time elapsed between the $K^{-}$entrance in a high density gas and its subsequent capture/absorption 
Table 1. $K^{-} N$ interaction channels and their corresponding branching ratios [12-16] (* channels which are not producing charged pions).

\begin{tabular}{|c|c|c|}
\hline Interaction & Channel & Branching ratio \\
\hline$K^{-} p$ & $\Sigma^{-} \pi^{+} \rightarrow\left(n \pi^{-}\right) \pi^{+}$ & $15.92 \%$ \\
\hline$K^{-} p$ & $\Sigma^{+} \pi^{-} \rightarrow\left(p \pi^{0}\right) \pi^{-}$ & $18.56 \%$ \\
\hline$K^{-} p$ & $\Sigma^{+} \pi^{-} \rightarrow\left(n \pi^{+}\right) \pi^{-}$ & $17.12 \%$ \\
\hline$K^{-} p$ & $\Sigma^{0} \pi^{0} \rightarrow(\Lambda \gamma) \pi^{0} \rightarrow\left(\left(p \pi^{-}\right) \gamma\right) \pi^{0}$ & $15.52 \%$ \\
\hline$K^{-} p$ & $\Sigma^{0} \pi^{0} \rightarrow(\Lambda \gamma) \pi^{0} \rightarrow\left(\left(n \pi^{0}\right) \gamma\right) \pi^{0}$ & $8.72 \% *$ \\
\hline$K^{-} p$ & $\Lambda \pi^{0} \rightarrow\left(p \pi^{-}\right) \pi^{0}$ & $2.66 \%$ \\
\hline$K^{-} p$ & $\Lambda \pi^{0} \rightarrow\left(n \pi^{0}\right) \pi^{0}$ & $1.54 \% *$ \\
\hline$K^{-} n$ & $\Sigma^{-} \pi^{0} \rightarrow\left(n \pi^{-}\right) \pi^{0}$ & $4.32 \%$ \\
\hline$K^{-} n$ & $\Sigma^{0} \pi^{-} \rightarrow(\Lambda \gamma) \pi^{-} \rightarrow\left(\left(p \pi^{-}\right) \gamma\right) \pi^{-}$ & $2.76 \%$ \\
\hline$K^{-} n$ & $\Sigma^{0} \pi^{-} \rightarrow(\Lambda \gamma) \pi^{-} \rightarrow\left(\left(n \pi^{0}\right) \gamma\right) \pi^{-}$ & $1.56 \%$ \\
\hline$K^{-} n$ & $\Lambda \pi^{-} \rightarrow\left(p \pi^{-}\right) \pi^{-}$ & $7.27 \%$ \\
\hline$K^{-} n$ & $\Lambda \pi^{-} \rightarrow\left(n \pi^{0}\right) \pi^{-}$ & $4.09 \%$ \\
\hline
\end{tabular}

by a deuterium atom has a typical range from 3 to $7 \mathrm{~ns}$ and is driven by the number of collisions required to transfer most of its kinetic energy to the gas atoms. This class of events is easily distinguishable from those related to a $K^{-}$stopped in the solid elements of the setup, followed by X-ray transitions and nuclear absorption. Hence, the detector proposed as second level trigger (scintillator barrels surrounding the setup) takes advantage of the high probability $(\simeq 90 \%)$ for the production of charged pions following the $K^{-}$absorption by deuterium nucleus, as arises from the study of the production channels and corresponding branching ratios (see table 1 [12-16]).

\section{Monte Carlo simulations}

\subsection{Full setup Monte Carlo simulations}

A detailed Monte Carlo (MC) simulation was done in order to check eventual constraints to be fulfilled and to obtain the gain factor in the signal over background ratio. The SIDDHARTA-2 experimental setup technical drawing is presented in figure 1; the setup consists in a cryogenic target filled with high density deuterium gas at $20 \mathrm{~K}$, surrounded by an array of 144 silicon drift detectors (SDD) meant to detect the X-rays emitted by the kaonic atoms. The SDD array is cooled down to $\sim 150 \mathrm{~K}$ inside a vacuum chamber $(\simeq 160 \mathrm{mbar})$, to reduce the thermal noise of the silicon. The kaons entering the target are tagged by two plastic scintillators (kaon trigger), placed just below and above the interaction point, to take advantage of the back-to-back $\Phi$ decay into $K^{+} K^{-}$. The cylindrical vacuum target chamber, except for the upper side, will be surrounded by the T2L scintillators. The T2L consists of a set of 12 rectangular scintillators each coupled at both sides to Photomultipliers (PMT) through ad-hoc shaped light guides. Due to the presence of the 


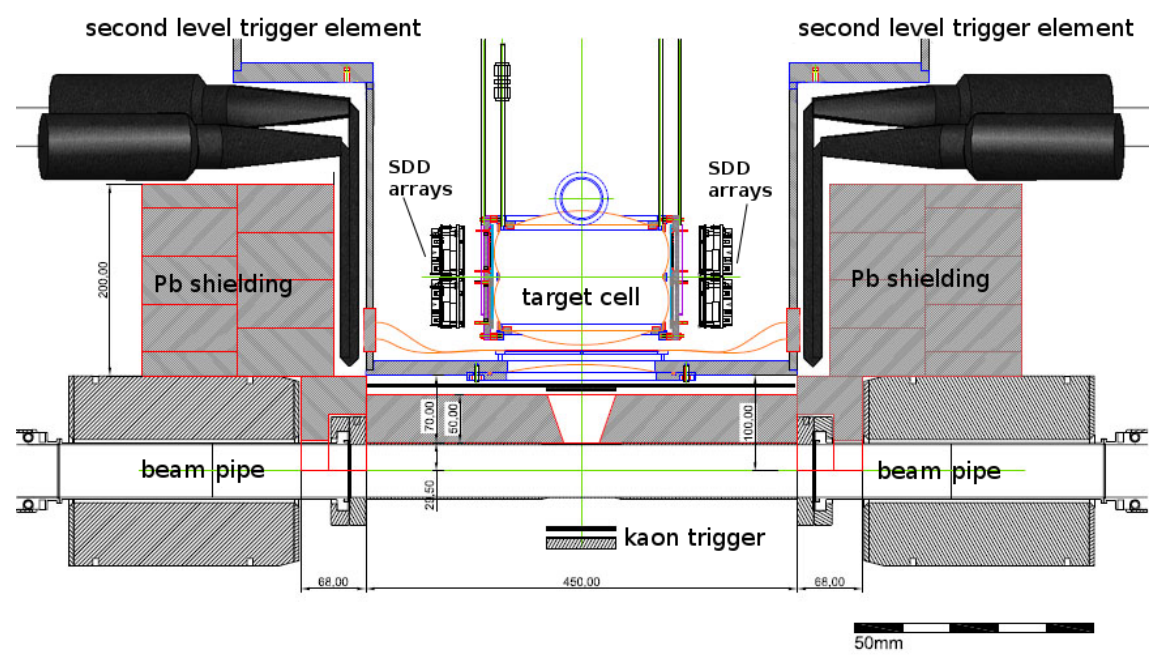

Figure 1. A detailed technical drawing of the SIDDHARTA-2 experimental setup sideview section (details can be found in text and in [11]). The elements of the second level trigger (T2L), placed around the vacuum chamber, are showed in black.
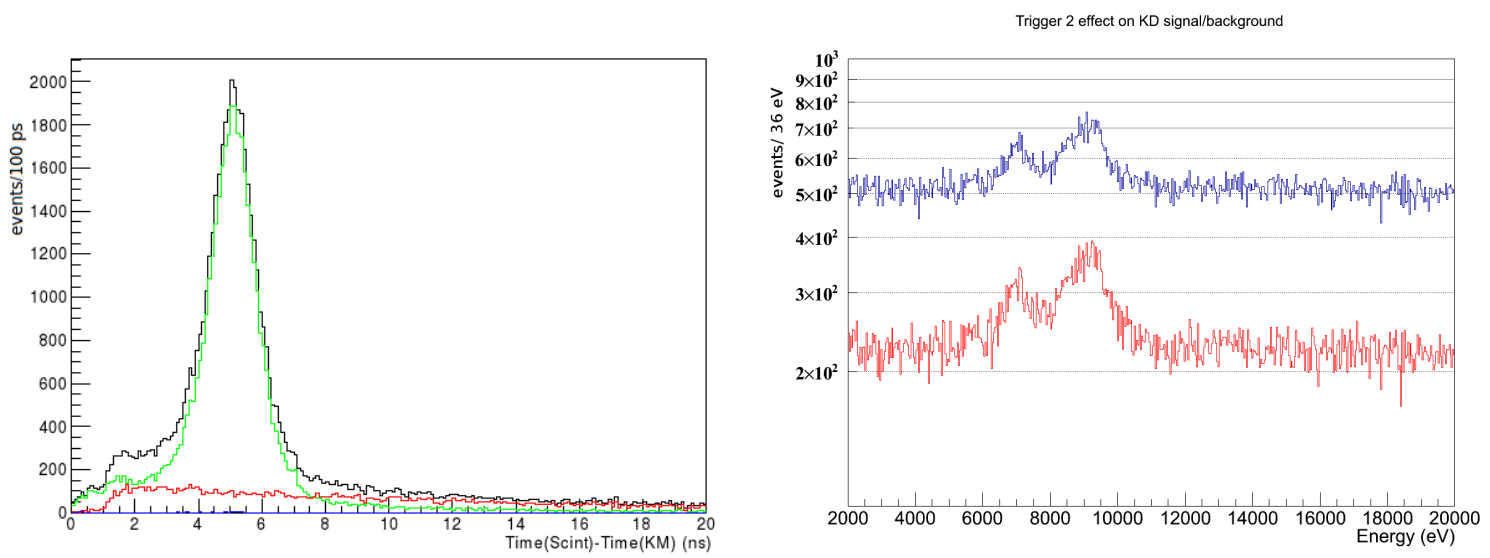

Figure 2. Left: GEANT4 simulated time spectrum for $2 \times 10^{6} \Phi$ decays of a scintillator placed outside the vacuum chamber. The main peak corresponds to particles produced by the $K^{-}$absorption by a gas nucleus. Right: simulated KD X-ray spectrum (in a logaritmic scale) using the T2L (red) and without it (blue).

external shielding, a complex geometry of the scintillators, light guides and PMTs has to be used, in order to fit in the free space available around the target cell, as shown in figure 1.

The T2L system is meant to detect the charged pions produced in the kaon-nucleon absorption reactions listed in table 1 . The events we are interested in are those in which kaons are stopped in the gas while all the others, as those where the kaons are stopped in the target cell window, have to be rejected. The separation can be done using the arrival time of the charged particles. The results of the simulated time spectra for $2 \times 10^{6} \Phi$ decays are shown in the left part of figure 2 , where the main peak corresponds to particles produced by the $K^{-}$absorption by a gas nucleus.

In the left part of figure 2 the black line represents the time difference between a trigger signal $\left(K^{+} K^{-}\right.$produced by the $\Phi$ decay in DA $\Phi N E$ in a back-to-back configuration) and a $\pi^{ \pm}$signal 
on the scintillator. The green peak shows the subset of events in which a $K^{+}$crosses the bottom side of the kaon detector (implying a $K^{-}$reached the target) while the red one shows the ones in which a $K^{-}$crosses the bottom side of the kaon detector (the $K^{+}$is then stopped in the target or in the walls). In blue, a spectrum where the bottom kaon detector detects neither a $K^{+}$nor a $K^{-}$ is shown. In order to separate events of $K^{-}$absorbed in gas from the unwanted ones, in particular those belonging to the left shoulder in the time spectrum in the left part of figure 2 , the required time resolution should be below $1 \mathrm{~ns}$ (FWHM). The simulation shows that using this selection a gain factor of 1.8 in signal over background ratio can be achieved. The effect of the good events selection of the final X-ray spectrum is shown in the right side of figure 2, where the red line represents the simulated energy spectrum of KD with T2L included, while the blue line is the simulated energy spectrum without T2L. Event axis is in a logaritmic scale. A further gain factor of $\sim 1.3$ can be obtained by topologic correlation between the scintillators and the X-ray detectors.

\subsection{Second level trigger dedicated study}

To optimize the T2L response, a separate MC study has been performed. The goal of the study was obtaining the best set of geometrical parameters granting a high number of detected photons as well as a minimum signal time spread. Typically, minimum ionizing particles deposit between 1.8 and $3 \mathrm{MeV}$ in $1 \mathrm{~cm}$ of scintillator, resulting in a light yield of 18000 to 30000 photons. The energy distribution follows the photon emission spectra of the scintillator while the decay time for the excited state of the scintillator was set to $2 \mathrm{~ns}$ and the attenuation length to $400 \mathrm{~cm}$ [17]. As long as a photon stays alive, its trajectory is mainly determined by the feature of the reflection surfaces. The processes involving optical photons at the boundaries between mediums are treated by GEANT4 with dedicated libraries, containing sets of parameters for mechanically polished, roughcut and chemically etched surfaces, combined with typical reflector materials like Aluminum, air, and dielectric reflectors [18]. According to the simulation, a polished or etched surface will give comparable time response, with a largest flux of photons reaching the PMT cathode in less than $1 \mathrm{~ns}$, while a rough cut surface will reach the maximum signal in no less than $2 \mathrm{~ns}$. Taking advantage of the large number of initial photons, our design optimisation procedure concerns mostly the time compaction of the output photon pulse, rather than a very high collection efficiency. As no significant variation in the light collection efficiency between various reflectors was reported in [18], the wrapping was performed with standard aluminized foils. To optimize the light transfer between scintillator bars and light guides, the width of the optical contact was varied around the prism height by plus $20 \mathrm{~mm}$ to minus $10 \mathrm{~mm}$. We found that increasing the width of the contact does not increase the number of photons that reach the far PMT, while reducing the area the photon number starts to decrease linearly. The overall result of simulated photon transport gives an average fraction of $7 \%$ reaching the PMT cathode placed on the long light guide, while $12 \%$ of photons are arriving at the PMT placed on the short one. To subsequently estimate the time resolution of the output signal, we generated $1 \times 10^{5}$ photo-electrons for each photon reaching the cathode. This value accounted for the cathode quantum efficiency and the PMT operation at minimum gain. The timing of each photo-electron is further randomized, according to the transit time spread of $0.28 \mathrm{~ns}$ and to the PMT rise time of $2 \mathrm{~ns}$ (parameters taken from the Hamamatsu data sheet [19]). In order to reduce the spread in the photon path length, various longitudinal segmentations of the scintillator and light guide were studied. The maximum number of stripes was limited to three, because 


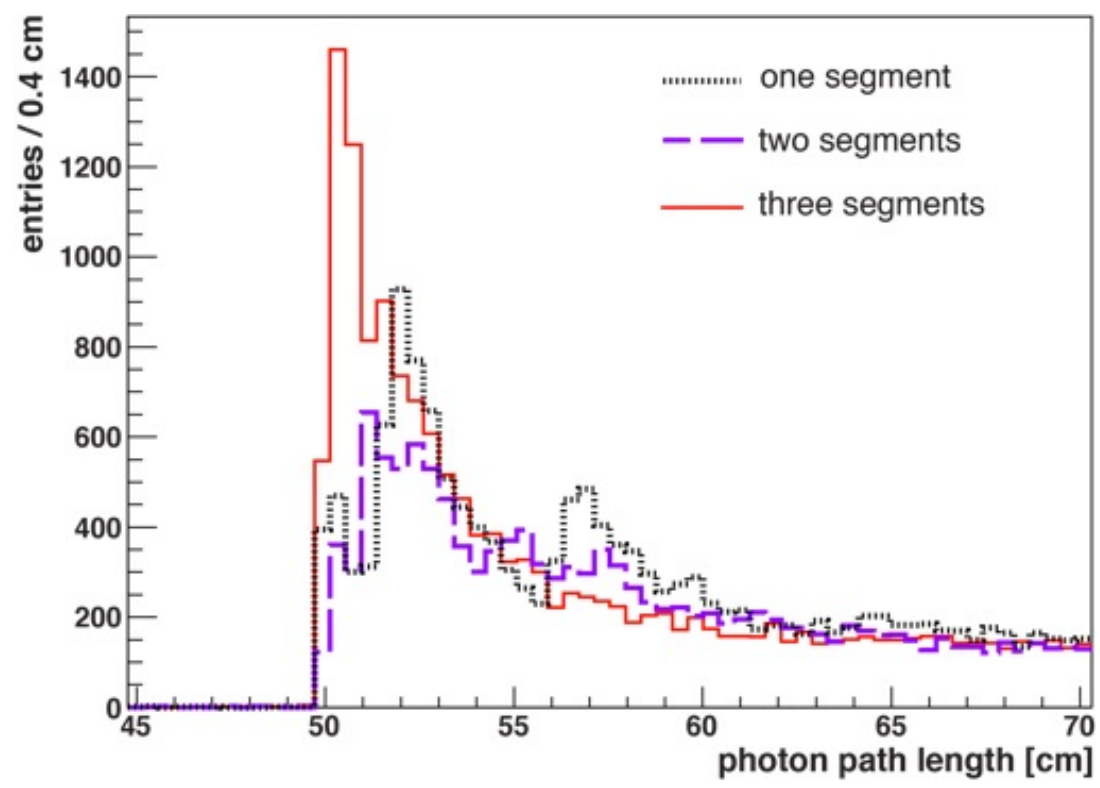

Figure 3. Path lenght distribution for photons reaching the far cathode, corresponding to 10 charged particles hitting the center of the scintillator. Black dotted line shows the distribution for one segment, violet dashed line for two segments and red line for three segments of the scintillators and light guides.

the simulation shows that beyond this level the path length spread does not contribute significantly to the overall time response of the system (figure 3), while higher segmentation would introduce alignment problems related to machining tolerance.

Finaly, a prototype has been built following the MC specifications. The detailed description and the drawing (in figure 4) of the prototype can be found in the next section.

\section{Second level trigger prototype test}

In order to test the performance of the proposed T2L system, the efficiency and the time resolution of a prototype were measured in the $\pi M-1$ beamline of the Paul Scherrer Institute of Villigen in Switzerland (PSI) [20]. The drawing of the prototype is shown in figure 4. A $(260 \times 110 \times$ 10) $\mathrm{mm}^{3}$ plastic scintillator (ELJEN TECHNOLOGY, EJ-200) segmented into 3 equal stripes is read out at both ends by 2 Hamamatsu R10533 PMTs. The PMTs were carefully chosen as the best compromise between area (matching the scintillator one), transit time spread, gain and noise. The double read-out is necessary for our purposes because, in addition to a better (mean time) resolution, the detector could provide an information on the hit position (with an average error of $2-3 \mathrm{~cm}$ ), by measuring the time difference between the 2 PMTs. Since in SIDDHARTA-2 the scintillators can be accessed only on one side, in a narrow, transverse angle, a complex system of mirrors and light guides ensures the photon extraction. Basically, each scintillator stripe is lined-up with a corresponding light guide (PMMA polivar ${ }^{\circledR}$ ), to which it has a lateral optical contact at one end. Photon transportation from scintillator to light guide (and collector) is ensured by the $45^{\circ}$ polished cuts on both elements, behaving like reflecting surfaces (total reflection angle is $\sim 42^{\circ}$ ). In addition, aluminized mirrors were installed to recover the refracted photons propagated at large 


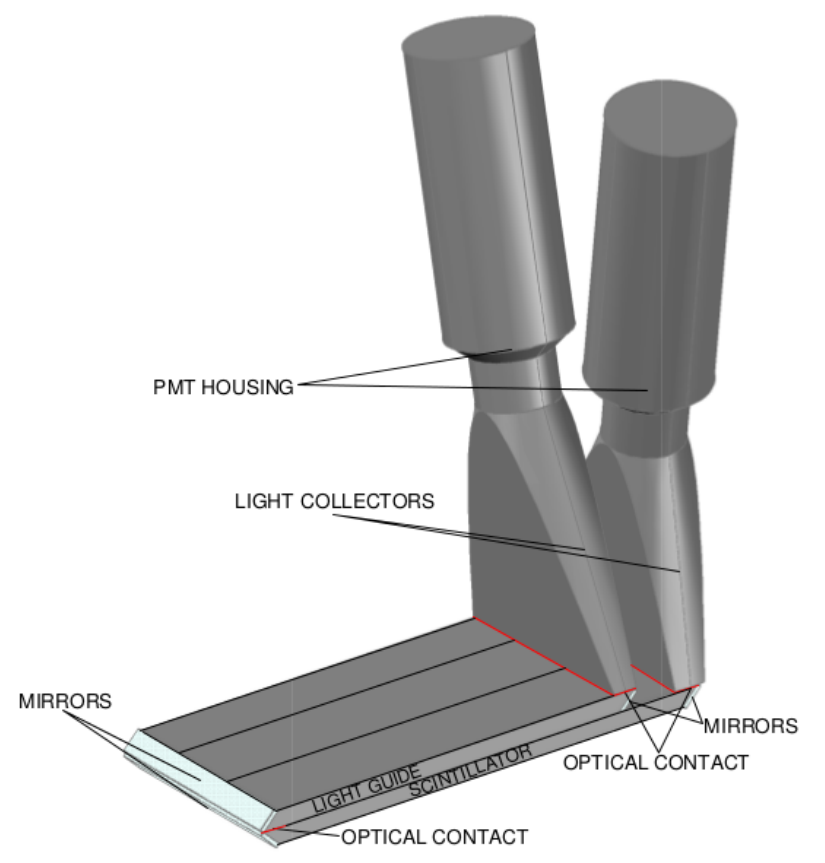

Figure 4. The T2L prototype scheme; 3 stripes of scintillator are coupled each with a stripe of light guide by an optical grease. Light collectors leading to photomultipliers (PMTs) are glued both on scintillators and light guides by optical glue. At the ends of the $45^{\circ}$ cuts of the scintillators and light guides there are aluminized mirrors placed to lead the photons towards the PMTs.

angles. The contact between the light guide and the scintillator was made with optical grease, while collectors are glued to the scintillator (SHORT) and the light guide (LONG). Except for the contact area, all stripes and collectors were individually wrapped in aluminized mylar. The whole detector was made light-tight by blacktape wrapping. The collectors were also made from PMMA; their optical contact matches the cathode area, while side surfaces are flat and conical, with $-3^{\circ}$ and $+28^{\circ}$ respectively, ensuring optimal light transfer to PMT. Accordingly to the chosen optics, the scintillating light collection follows 2 paths, independently measured: a short one (SHORT), in which the photons are reflected by a single mirror directly onto the collector, coupled to a PMT, and a long one (LONG), passing trough 3 mirrors, a light guide and a collector before reaching the other PMT cathode.

The tests of the prototype were made at the PSI using the $170 \mathrm{MeV} / \mathrm{c}$ momentum pions available at the $\pi M-1$ beamline. The experimental setup (not in scale) is shown in figure 5 . The incident beam particles were detected by two $(20 \times 10 \times 10) \mathrm{mm}^{3}$ fast plastic scintillators F1 and F2 before entering a box containing the T2L prototype. The trigger was given by the coincidence between F1, F2 and the Radio-Frequency(RF) clock of the $\pi M-1$ beamline. A position scan of the prototype was performed measuring 9 regions distributed over a $3 \times 3$ matrix along the $\mathrm{x}$ and $y$ directions of the scintillator surface. The beam spot and therefore the coordinate accuracy were defined by $\mathrm{F} 1$ and $\mathrm{F} 2$ sizes $(20 \mathrm{~mm} \times 10 \mathrm{~mm})$. 


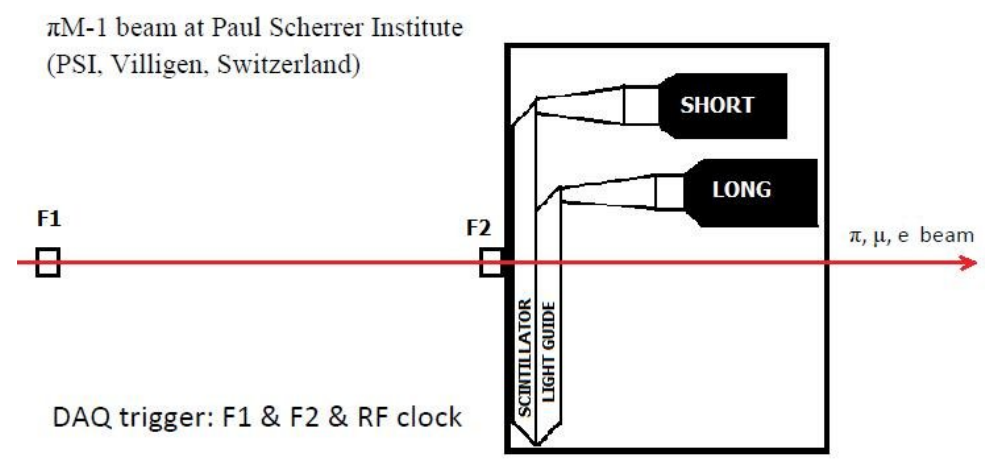

Figure 5. Experimental setup scheme. The particles are detected by two $(20 \times 10 \times 10) \mathrm{mm}^{3}$ fast plastic scintillators $\mathrm{F} 1$ and $\mathrm{F} 2$ before entering a box containing the $\mathrm{T} 2 \mathrm{~L}$ prototype scintillator. The system was triggered by the coincidence between F1, F2 and the RF clock of the $\pi M-1$ beamline.

\section{Beam test results}

\subsection{DAQ chain}

The DAQ chain used for the measurements was arranged as follows: the analog signals of the scintillators were splitted in order to collect both the QDC and the TDC information. ORTEC 935 CFD and CAEN V1190B modules were used for the timing measurement, while the QDC information was obtained using a CAEN V792A/C module. The trigger was given by the F1\&F2\&RF triple coincidence. The data from each scintillator were acquired and stored individually.

\subsection{Particles identification and events selection}

In order to obtain individual results for different particles, pions, muons and electrons have to be identified. A 2D plot of the hit time on the 2 fast scintillators (F1 and F2), referenced by the RF clock, allowed a clean separation of the 3 components by their time-of-flight. The plot is shown in figure 6, where pions, muons and electrons selection cuts are overimposed.

\subsection{Time resolution results}

The time resolution analysis was done for the $170 \mathrm{MeV} / \mathrm{c}$ momentum pions, muons and electrons, representing the mean value of $\bar{K} N$ absorption products momentum. TDC spectra of LONG, SHORT (again, with respect to the $\pi$-M1 RF) and their Mean Time (MT) ((LONG + SHORT)/2) were fitted with Gauss functions for each one of the 9 measured distributions. As an example, the fitted MT spectrum for the central region $(x=13 \mathrm{~cm}, y=5.5 \mathrm{~cm})$ of the scintillator surface is shown in figure 7. The same analysis was applied to all the 9 measurement regions across the scintillator surface. The time resolutions, with statistic and systematic errors respectively, are presented in figure 8 where results are reported on each position for pions (red), muons (green) and electrons (blue). The systematic errors were evaluated by varying the selection areas and recording the corresponding variations in the resolution. 


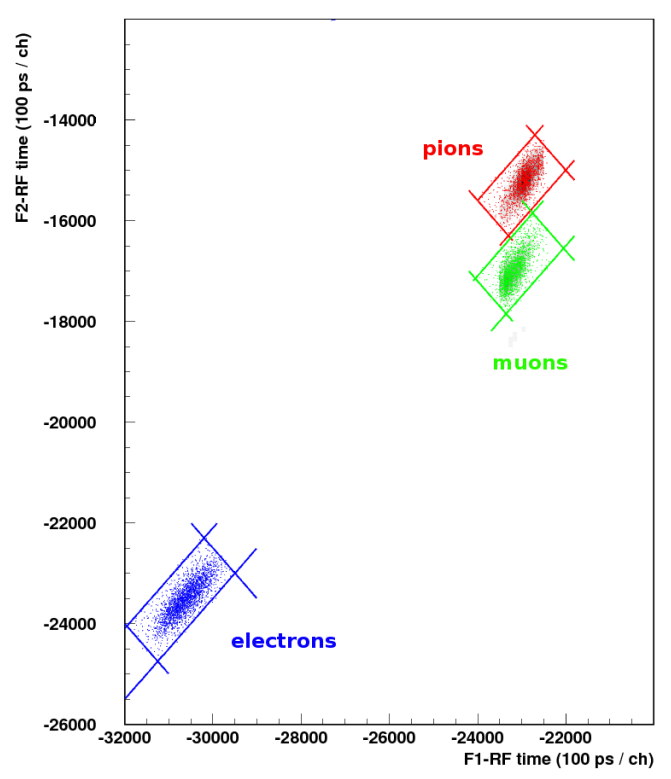

Figure 6. 2D graph of the TDC signals of F1 and F2. The selection plot for the $170 \mathrm{MeV} / \mathrm{c}$ momentum beam; pions (red), muons (green) and electrons (blue) clusters are clearly visible.
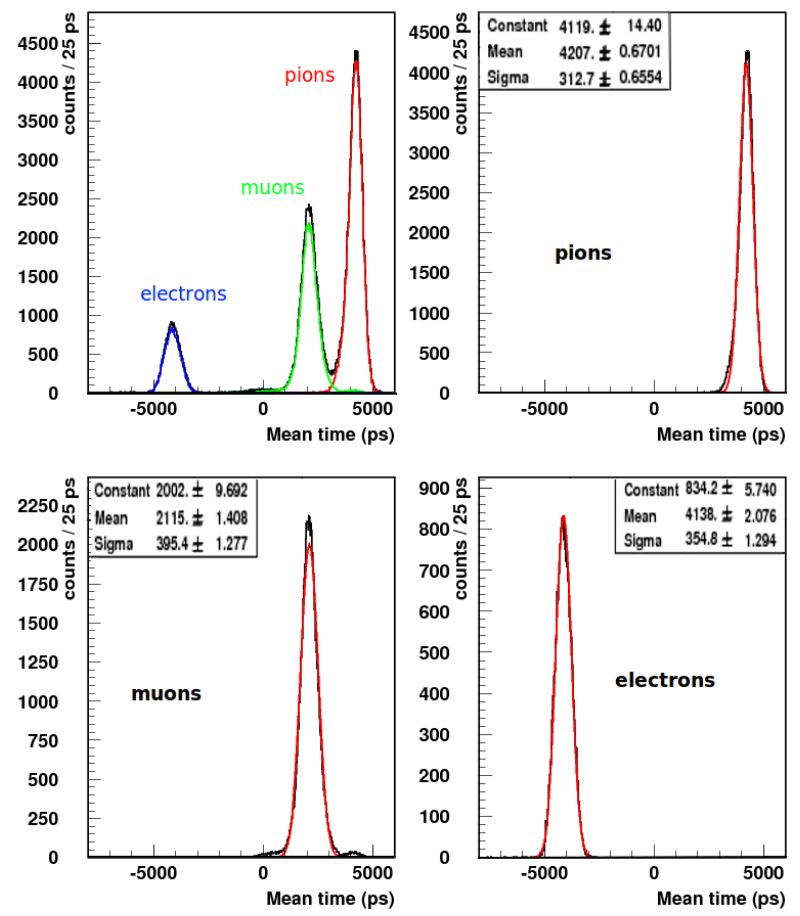

Figure 7. The fitted MT spectra for the central region of the scintillator surface $(x=13 \mathrm{~cm}, y=5.5 \mathrm{~cm})$; spectra of selected pions (red), muons (green) and electrons (blue) are shown in the upper left corner. Fitted distributions are presented in the upper right corner for pions, in the down left corner for muons and in the down right corner for electrons. 


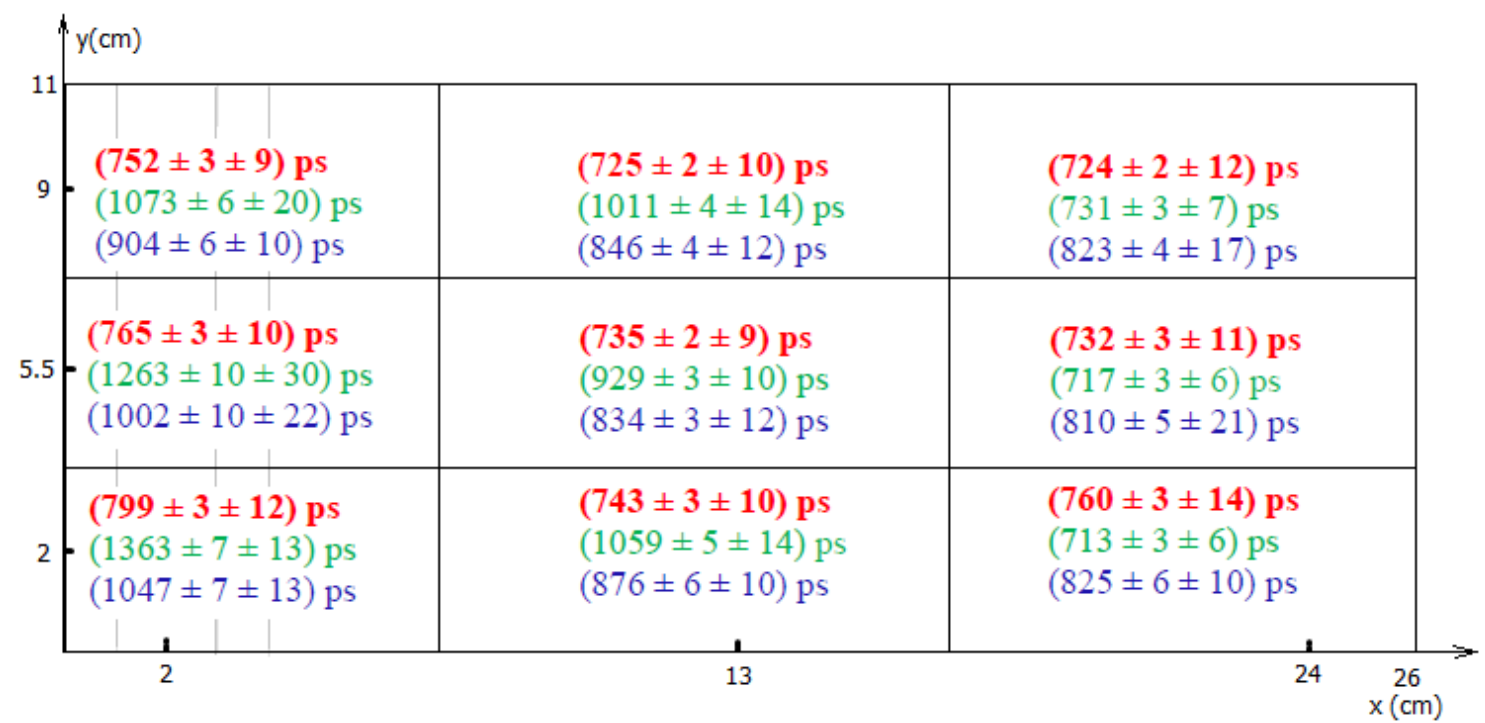

Figure 8. Measured mean time resolutions (FWHM) for $170 \mathrm{MeV} / \mathrm{c}$ momentum pions (red), muons (green) and electrons (blue).

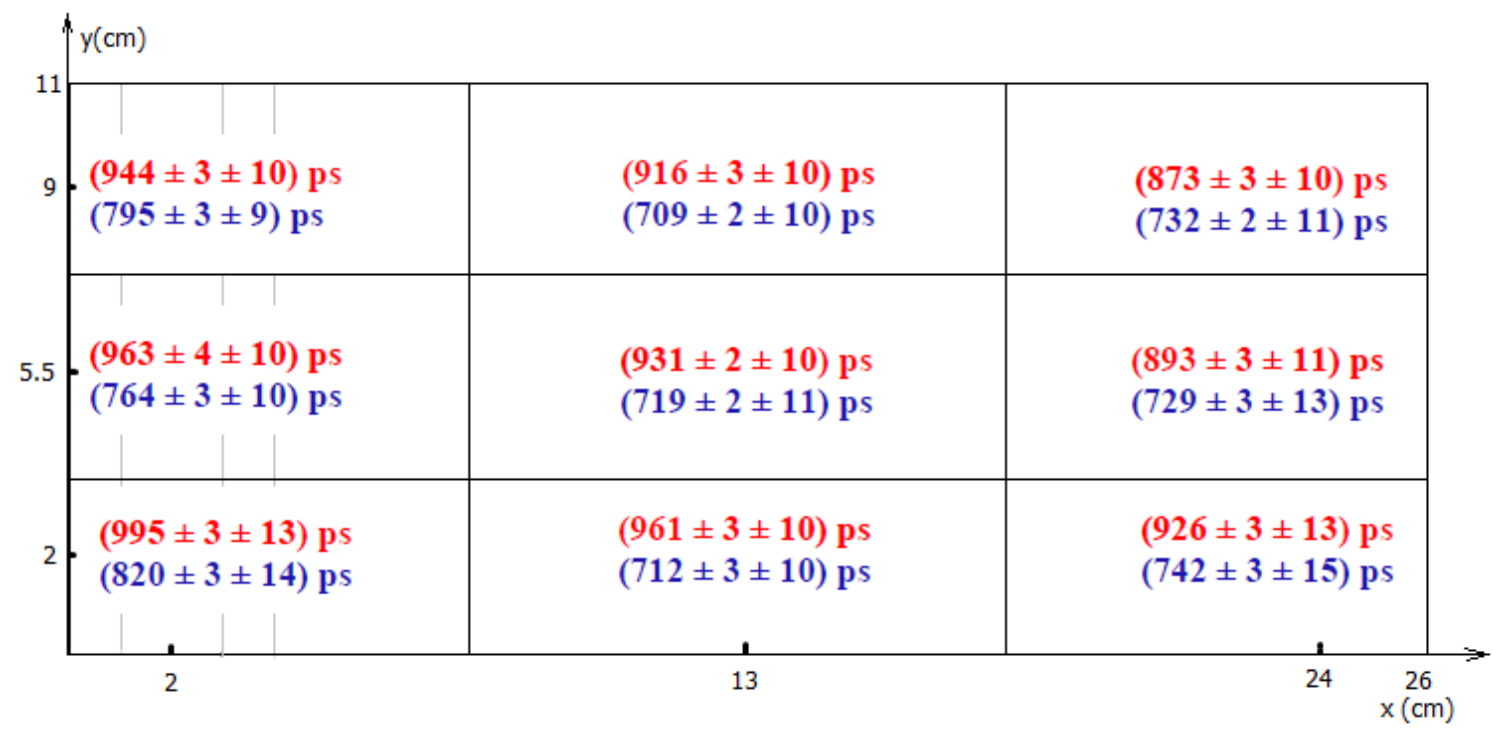

Figure 9. Measured time resolutions (FWHM) for the LONG (red) and SHORT (blue) for the $170 \mathrm{MeV} / \mathrm{c}$ momentum pions.

In order to compare the performances of the LONG and SHORT scintillators, the individual measured resolutions for pions are presented in figure 9. The results show that with the introduction of the multi reflection light guide (LONG), the FWHM is worsened by $\simeq 25 \%$. The overall time resolution (FWHM) for $170 \mathrm{MeV} / \mathrm{c}$ momentum pions (MT) was measured to be $(746 \pm 53) \mathrm{ps}$, where the error is taken as the maximum deviation from the mean value; the observed small variations corresponding to the different positions are in the order of $\sim 4.5 \%$. 


\begin{tabular}{|c|c|c|c|}
\hline \multicolumn{4}{|c|}{$y(\mathrm{~cm})$} \\
\hline 9 & $\begin{array}{l}(\mathbf{9 4 . 6} \pm \mathbf{0 . 6} \pm \mathbf{0 . 1}) \mathbf{\%} \\
(95.91 \pm 0.78 \pm 0.04) \% \\
(99.33 \pm 1.34 \pm 0.01) \%\end{array}$ & $\begin{array}{l}(96.03 \pm \mathbf{0 . 5 1} \pm \mathbf{0 . 0 8}) \% \\
(97.00 \pm 0.64 \pm 0.05) \% \\
(99.876 \pm 1.111 \pm 0.006) \%\end{array}$ & $\begin{array}{l}(\mathbf{9 6 . 5 0} \pm \mathbf{0 . 5 5} \pm \mathbf{0 . 0 7}) \% \\
(96.42 \pm 0.70 \pm 0.03) \% \\
(99.891 \pm 1.207 \pm 0.02) \%\end{array}$ \\
\hline .5 & $\begin{array}{l}(\mathbf{9 6 . 3 9} \pm \mathbf{0 . 6 2} \pm \mathbf{0 . 0 7}) \% \\
(97.86 \pm 0.80 \pm 0.02) \% \\
(99.973 \pm 1.338 \pm 0.001) \%\end{array}$ & $\begin{array}{l}(\mathbf{9 6 . 6 8} \pm \mathbf{0 . 3 7} \pm \mathbf{0 . 0 7}) \% \\
(97.82 \pm 0.48 \pm 0.04) \% \\
(99.960 \pm 0.816 \pm 0.001) \%\end{array}$ & $\begin{array}{l}\mathbf{( 9 6 . 1 9} \pm \mathbf{0 . 6 1} \pm \mathbf{0 . 0 7}) \% \\
(96.94 \pm 0.79 \pm 0.03) \% \\
(99.98 \pm 1.40 \pm 0.01) \%\end{array}$ \\
\hline 2 & $\begin{array}{l}\mathbf{( 9 6 . 1 1} \pm \mathbf{0 . 6 1} \pm \mathbf{0 . 0 7}) \% \\
(97.11 \pm 0.78 \pm 0.06) \% \\
(99.919 \pm 1.427 \pm 0.009) \%\end{array}$ & $\begin{array}{l}\mathbf{( 9 3 . 5} \pm \mathbf{0 . 6} \pm \mathbf{0 . 1}) \mathbf{\%} \\
(93.9 \pm 0.8 \pm 0.3) \% \\
(98.0 \pm 1.3 \pm 0.2) \%\end{array}$ & $\begin{array}{l}\mathbf{( 9 5 . 9 6} \pm \mathbf{0 . 6 1} \pm \mathbf{0 . 0 8}) \% \\
(96.00 \pm 0.77 \pm 0.12) \% \\
(99.93 \pm 1.44 \pm 0.02) \%\end{array}$ \\
\hline & 2 & 13 & 24 \\
\hline
\end{tabular}

Figure 10. Measured efficiencies (MT) for the $170 \mathrm{MeV} / \mathrm{c}$ momentum pions (red) muons (green) and electrons (blue).

\subsection{Efficiency results}

The efficiency of the T2L prototype was checked with a procedure similar to the one used for the time resolution. The same F1\&F2 selections were applied for pions, muons and electrons. For each kind of particle the efficiency is defined as the ratio between the number of particles detected by the T2L prototype over the ones detected by F1\&F2. The results for pions (red), muons (green) and electrons (blue) are presented in figure 10 for each measuring region. The overall efficiency for $170 \mathrm{MeV} / \mathrm{c}$ momentum pions (MT) was measured to be $96 \pm 2 \%$.

\section{Higher momentum test results}

Although the main working regime for the proposed detector is centered on the reported momentum range, a "worst-case scenario" concerning the upper tail of pion momentum distribution $(255 \mathrm{MeV} / \mathrm{c})$ was investigated too. We will not provide here the full data analysis but rather the two average results, indicating a time resolution of $\sim 880 \mathrm{ps}$ and an efficiency of $\sim 94.5 \%$.

\section{Conclusions}

The time resolutions and the efficiencies for a non-conventional detector based on scintillators coupled to a prism reflector light guide and equipped with 4 mirrors, were measured for $170 \mathrm{MeV} / \mathrm{c}$ momentum pions, muons and electrons at the $\pi M-1$ beamline of the PSI. For the pion beam the measured Mean Time resolution is $(746 \pm 53)$ ps FWHM, while the measured efficiency is $(96 \pm 2) \%$. Small variations, in the order of $\sim 4.5 \%$, were found on the time resolution for different position of the scintillator surface. Since in the SIDDHARTA-2 experiment such small variations do not represent a source of possible pion misidentification, the prototype proved to be an excellent solution for the second level trigger for a successful measurement of kaonic deuterium. 


\section{Acknowledgments}

Part of this work was supported by the European Community-Research Infrastructure Integrating Activity "Study of Strongly Interacting Matter" (HadronPhysics2, Grant Agreement No. 227431 and HadronPhysics3, Grant Agreement No. 283286) under the Seventh Framework Programme of EU. We thank to the PSI staff and in particular to Dr. Konrad Deiters and the whole $\pi \mathrm{M}-1$ line team for the excellent working conditions and support. We would also like to thank to Aldo Orlandi for his help in building the prototype.

\section{References}

[1] SIDDHARTA collaboration, M. Bazzi et al., A New Measurement of Kaonic Hydrogen X rays, Phys. Lett. B 704 (2011) 113 [arXiv: 1105.3090].

[2] SIDDHARTA collaboration, M. Bazzi et al., Kaonic hydrogen X-ray measurement in SIDDHARTA, Nuc. Phys. A 881 (2012) 88.

[3] SIDDHARTA collaboration, M. Bazzi et al., First measurement of kaonic helium-3 X-rays, Phys. Lett. B 697 (2011) 199 [arXiv: 1010.4631].

[4] SIDDHARTA collaboration, M. Bazzi et al., Measurements of the strong-interaction widths of the kaonic 3He and 4He 2p levels, Phys. Lett. B 714 (2012) 40 [arXiv: 1205.0640].

[5] D. Gazda and J. Mares, Calculations of $K^{-}$nuclear quasi-bound states based on chiral meson-baryon amplitudes, Nuc. Phys. A 881 (2012) 159.

[6] Y. Ikeda, T. Hyodo and W. Weise, Chiral SU(3) theory of anikaon-nucleon interactions with improved treshold constraints, Nuc. Phys. A 881 (2012) 98.

[7] E. Friedman and A. Gal, Kaonic atoms and in-medium K-N amplitudes, Nuc. Phys. A 881 (2012) 150.

[8] S. Deser, M. L. Goldberger, K. Baumann and W. Thirring, Energy level displacements in $\pi$ mesonic atoms, Phys. Rev. 96 (1954) 744.

[9] SIDDHARTA collaboration, M. Bazzi et al., Preliminary study of kaonic deuterium X-rays by the SIDDHARTA experiment at DAФNE, Nuc. Phys. A 907 (2013) 69.

[10] M. Faber et al., Energy Level Displacement of Excited np State of Kaonic Deuterium In Faddeev Equation Approach, Phys. Rev. C 84 (2011) 064314 [arXiv: 1012.3933].

[11] SIDDHARTA-2 collaboration, Proposal of Laboratori Nazionali di Frascati of INFN, The upgrade of SIDDHARTA apparatus for an enriched scientific case (2010).

[12] Particle Data Group collaboration, J. Beringer et al., Review of Particle Physics (RPP), Phys. Rev. D 86 (2012) 010001.

[13] GEANT4 collaboration, S. Agostinelli et al., GEANT4: A Simulation toolkit, Nucl. Instrum. Meth. A 506 (2003) 250.

[14] C. Vander Velde-Wilquet, J. Sacton, J. Wickens, D. Tovee and D. Davis, Determination of the Branching Fractions for $\mathrm{K}^{-}$Meson Absorption at Rest in Carbon Nuclei, Nuovo Cim. A 39 (1977) 538.

[15] C. Vander Velde-Wilquet et al., $K^{-}$-Meson Absorptions at Rest in Carbon Nuclei Leading to Final States Containing a Lambda Hyperon, Nuovo Cim. A 38 (1977) 178. 
[16] C. Vander Velde-Wilquet et al., The Conversion Probability and Emission Ratio of Charged Sigma Hyperons Following K ${ }^{-}$-Meson Capture at Rest in Carbon, Nucl. Phys. A 241 (1975) 511.

[17] http://www.eljentechnology.com/.

[18] M. Janecek and W.W. Moses, Simulating Scintillator Light Collection Using Measured Optical Reflectance, IEEE T. Nucl. Sci. 57 (2010) 964.

[19] Hamamatsu Photonics K.K., Photomultiplier tubes, Basics and Applications.

[20] http://aea.web.psi.ch/beam2lines/beam_pim1.html. 